\title{
ANÁLISIS ECONÓMICO DE LA TELEFONÍA MÓVIL EN EL PERÚ
}

\author{
PERU SUYUPI TELEFONIA MOVIL NISQA QULLQICHAKUY \\ CH'UYMAPAYMANTA
}

\section{ECONOMIC ANALYSIS OF MOBILE TELEPHONY IN PERU}

\section{Marilia S. Peceros Valencia ${ }^{(1)}$}

Ch'uyanchakuy: Analisis Economico nisqa Telefonia Movil khuskiypi Peru suyupi imaynas chay derechukunas mercado de telecomunicaciones nisqapi chinpuswan. Peru Suyupi khuskisaq, kay suyupi privatizacion nisqa huk hinalla, huk empresa nisqallaman concesion nisqata kunku, cahy hinaqa manan huk empresakunaman haykuta munankuchu, chaymi kallparichin monopolio nisqata chunka hukniyuq wataman, chay nisqapi hina iskay waranqa chunka kinsayuq watapi imaymana leykunata kuranku huk empresakuna nisqa haykunampaq, monopolio empresakuna tukukunampaq.

Yuyaysapa simikuna: Qolqenmanta kamachikunku, Qollana llank'ay wasi.

Resumen: Utilizar el Análisis Económico en el estudio de la Telefonía Móvil en el Perú nos permite medir la eficacia del marco regulatorio en el mercado de telecomunicaciones y su impacto en el comportamiento de las operadoras. El área de estudio fue Perú porque el proceso de privatización fue particular, toda vez que la concesión se otorgó a una sola empresa, y se restringió el ingreso de una nueva operadora, situación que fortaleció a la empresa pionera y le permitió mantener el monopolio por 11 años, en esas circunstancias en el año 2013 se dictan una serie de normas que fortalecen la competencia, permiten el ingreso de nuevas operadoras, y erosionan la ventaja de la empresa pionera.

Palabras Clave: Telefonía Móvil, Análisis Económico del Derecho, Empresa Pionera

(1) Abogada por la Universidad San Antonio Abad de Cusco, egresada de la Maestría en Derecho y Economía por la Universidad de Buenos Aires - Argentina. Trabajó en el Área de Planificación de la empresa VIETTEL PERÚ S.A.C., cuarta operadora de Telecomunicaciones en Perú. Actualmente labora en la Unidad de Planeamiento y Desarrollo del Poder Judicial. 


\begin{abstract}
Using Economic Analysis in the study of Mobile Telephony in Peru allows us to measure the effectiveness of the regulatory framework in the telecommunications market and its impact on the behavior of operators. The study area was Peru because the privatization process was particular, since the concession was granted to only one company, and the entry of a new operator was restricted, a situation that strengthened the pioneer company and allowed it to maintain its monopoly for 11 years long, in those circumstances in 2013, a series of law regulations strengthen competition, allowed the entry of new operators, and erode the advantage of the pioneer company.
\end{abstract}

Key Words: Mobile Telephony, Economic Analysis of Law, Pioneer Company 


\section{Antecedentes}

Hacia inicios de los años noventa el estado peruano dirigía dos empresas que prestaban servicios de telecomunicaciones. La compañía Peruana de Teléfonos SA (en adelante CPT) la cual operaba con exclusividad el servicio de telefonía local fija en Lima Metropolitana y el Callao; y la empresa Entel Perú SA (en adelante Entel), se encargaba de los servicios portador larga distancia nacional e internacional, telefonía fija para todas las provincias del Perú, con excepción de Lima Metropolitana y el Callao.

En los años previos a la privatización, el teléfono era considerado un bien suntuario. Una línea telefónica podía llegar a costar USD 2500.00 en el mercado negro y tomaba un plazo de cinco años para obtenerla.

En 1993, la densidad telefónica - medida como el número de líneas por cada 100 habitantes-era de 2.9; mientras que países similares tenían una densidad promedio de 9.2 .

\begin{tabular}{|l|c|c|}
\multicolumn{2}{|c}{ Cuadro $N^{\circ} \mathbf{1}^{(\mathbf{2})}$ : Densidad y Accesibilidad telefónica en Perú, año 1994} \\
\hline & $\begin{array}{l}\text { DENSIDAD } \\
\text { TELEFÓNICA (1) }\end{array}$ & $\begin{array}{c}\text { ACCESIBILIDAD AL } \\
\text { TELÉFONO (2) }\end{array}$ \\
\hline Perú a nivel Nacional & 2,9 & 10,1 \\
\hline Bolivia & 3,0 & 11,0 \\
\hline Paraguay & 3,1 & 9,7 \\
\hline Ecuador & 5,3 & 19,7 \\
\hline Chile & 11,0 & 39,1 \\
\hline Colombia & 11,3 & 33,9 \\
\hline
\end{tabular}

Fuente: Universidad del Pacífico.

En junio de 1992 se creó el Comité Especial de Privatización del sector de Telecomunicaciones (Cepri - Telecom) que definió que la importancia de la privatización se centraba en adjudicar el servicio telefónico a un operador internacional que contara con la tecnología adecuada y con una presencia importante en el sector (CÁCERES BARRANTES, 2005).

Con fecha 15 de mayo de 1994, se publicó el Decreto Supremo № 011 94-TCC, el mismo que aprobó los contratos de concesión a celebrarse con ENTEL y CPT-con accionariado de Telefónica Internacional SA de España, el contrato de concesión para la prestación del Servicio Portador y el Servicio Telefónico Local y de Larga Distancia Nacional e Internacional.

(2) Libro Experiencias de Regulación en el Perú de Jorge Fernández - Baca (Editor). Universidad del Pacífico. Lima 2004. P. 254 
De la misma manera, el Estado peruano representado por el Ministerio de Transportes y Telecomunicaciones -MTC, firmó contrato de concesión para la prestación del servicio Portador y el servicio telefónico local en las ciudades de Lima y Callao.

En diciembre de 1994 se concretaron la fusión entre ENTEL y CPT, absorbiendo esta última a la primera, para posteriormente cambiar la denominación por Telefónica del Perú S.A.A.

En 1998, a través del Decreto Supremo N021-98/MTC, el Ministerio de Transportes y Comunicaciones dispuso la reducción del periodo de concurrencia de cinco (05) a cuatro (04). Ello debido a que se habrían cumplido las metas más importantes que motivaron el establecimiento de dicho periodo, como así lo señala el libro blanco de las telecomunicaciones.

El 29 de diciembre de 1993 fue publicada la Ley $N^{\circ}$ 26285, que señalaba iniciar el proceso de apertura del mercado de las telecomunicaciones hacia un régimen de libre competencia. Ciertamente, el referido proceso consistiría en introducir competencia en los diferentes segmentos del sector para la prestación de servicios de telecomunicaciones, promoviendo el ingreso de nuevas operadoras. Ello coincidió con la publicación de los lineamientos de la Política de Apertura del Mercado de las Telecomunicaciones en el Perú. Aprobados por Decreto Supremo $N^{\circ}$ 020-98/MTC, del 04 de agosto de 1998 en los cuales se regularon temas vinculados a la regulación de tarifas, concesiones, interconexión de acceso universal, libre competencia, entre otros.

\subsection{Ingreso de América Móvil}

El 10 de mayo de 2005, América Móvil adquirió una licencia PCS 1900 MHz para prestar servicios de internet, telefonía fija y movil en Perú bajo la marca Sercotel Perú. El 10 de agosto de ese mismo año, América Móvil anuncia la adquisición de TIM Perú, y el 11 de octubre lanza la marca Claro.

En abril de 2008, se convierte en la primera empresa dentro del país en lanzar el servicio de internet móvil 3G, usando la tecnología HSDPA en la banda de $850 \mathrm{MHz}$ a una velocidad de hasta 1,5 Mbits.

El 01 de octubre de 2010, la compañía se fusiona con la filial peruana de Teléfonos de México -Telmex. El director comercial de América Móvil, Rodrigo Arosemena, confirmó el inicio del proceso de adquisición. Claro absorbe la empresa así como sus productos, y las distribuye bajo su propia marca. 


\subsection{Ingreso de Viettel Perú SAC}

En el año 2011, Viettel se adjudicó la concesión de espectro del Bloque C de la Banda de $1900 \mathrm{MHz}$ para poder brindar el servicio de internet, telefonía fija y móvil en el país, convirtiéndose así en el cuarto operador móvil en el Perú.

Posteriormente, en agosto de 2012 se adjudicó espectro adicional en la banda de $900 \mathrm{MHz}$. Sin embargo, las operaciones comerciales de Viettel recién iniciaron formalmente el 26 de julio de 2014 bajo la marca comercial "Bitel". El retraso se debió a que la empresa enfrentó ciertas dificultades tales como la lentitud en la obtención de los permisos municipales requeridos para desplegar su infraestructura y constantes robos de la fibra óptica ya desplegada.

\subsection{Ingreso del Grupo Entel}

Las intenciones del Grupo Entel Chile de ingresar al mercado móvil peruano se hicieron conocidas cuando, en abril de 2013, se anunció la compra de los activos de Nextel Perú S.A. (en adelante, Nextel Perú) por parte del Grupo Entel Chile, grupo que ya contaba con presencia en el mercado de telecomunicaciones peruano, aunque de forma indirecta, por ser la matriz de Americatel Perú S.A. (en adelante, Americatel), operador enfocado en las comunicaciones de larga distancia. Dicha transacción se concretó el 20 de agosto de 2013, por un monto de US\$ 400 millones.

Paralelamente, en julio de 2014 el Grupo Entel Chile, a través de Americatel, ganó el Bloque B de la banda AWS, destinada a servicios móviles avanzados de alta velocidad $4 G$ vía tecnología LTE.

Posteriormente, el 25 de junio de 2014, mediante Resolución Viceministerial N³80-2014-MTC/03 se aprobó la transferencia de la asignación de este espectro a favor de Nextel Perú.

Si bien el Grupo Entel ya poseía los activos de Nextel Perú, mantuvieron el nombre comercial "Nextel" hasta el 10 de octubre de 2014, fecha en que lanzaron su nuevo nombre comercial "Entel Perú".

A pesar de que este acontecimiento no puede considerarse propiamente como el "ingreso de un nuevo operador móvil", pues Nextel Perú ya operaba en el mercado móvil desde 1998, sí es un hecho trascendental para el desempeño del mercado móvil, pues el cambio de grupo económico se tradujo en un notable cambio en la estrategia de este operador.

\subsection{Lanzamiento de la Marca "Tuenti" por Telefónica}

Telefónica brindaba todos sus servicios bajo una única marca comercial: "Movistar"; sin embargo, en octubre de 2014 lanzó una nueva marca comercial denominada 
"Tuenti". Esta nueva marca está enfocada en el segmento residencial prepago juvenil, es por ello que su estrategia se basa en promocionar una oferta sencilla a través de una lenguaje coloquial cercano al público joven.

\subsection{Ingreso de Virgin Movil Perú}

Inició sus operaciones el 22 de julio de 2016. Inicialmente su estrategia comercial fue proveer el servicio de telefonía móvil con tarjetas SIM sin contratos, adicionó a su esquema comercial la venta de equipos móviles a través de alianzas con tiendas por departamento y supermercados.

El servicio ofrecido es similar a su estrategia en otros países, tales como: AntiPlanes, que son paquetes que combinan datos, voz y sms, además de acceso a redes sociales (como Facebook y Whatsapp) los cuales tienen la ventaja de acumular las megas no usados para el siguiente mes.

Virgin Mobile Perú, operó durante 13 meses, con el objeto de ganar el $2 \%$ de mercado anual sin embargo, al tiempo de vender sus acciones sólo habría acumulado 100 Mil líneas, que equivale al $0.16 \%$ del mercado.

\subsection{Ingreso de Inkacell}

Esta nueva operadora móvil virtual compró los activos de Virgin Mobile Perú, y prosigue con el contrato que esta última contrajo con Movistar por el periodo de cinco años para el alquiler de infraestructura.

La estrategia comercial de este nuevo operador móvil virtual, es ofrecer precios más competitivos para efectuar recargas, con ello se podría efectuar una recarga por un costo de $0.50^{(3)}$ centavos de sol.

En cuanto a innovación, este nuevo operador móvil ofrece las recargas mediante los teléfonos públicos, lo que resulta novedoso toda vez que en el mercado peruano, los teléfonos públicos son utilizados sólo para hacer llamadas. Inkacell ha sido lanzado oficialmente en la quincena de octubre del 2017. Aunque el CEO de la empresa, ha manifestado en declaraciones a los medios de comunicación que las innovaciones tecnológicas llegarían a partir de la primera quincena del 2018. Lo cual a la fecha de redactado el artículo no se ha cumplido.

\subsection{Ingreso de Cuy Mobile, Dolphin y Farmagusta}

Según declaraciones al diario El Comercio, el viceministro de transportes y comunicaciones anunció que tras la salida de Virgin Mobile, tres nuevas operadoras

(3) Aproximadamente 0.15 centavos de dólar 
móviles virtuales harían su ingreso al mercado, siendo una de ellas de capitales peruanos (Cuy Móvil). Al ser estas Operadoras Móviles Virtuales-OMV- no poseen red, por lo que deben alquilar infraestructura de telecomunicaciones.

\section{La estrategia de ser pionera en el mercado de la telefonía movil.}

Las empresas que entran primero al mercado obtienen ventajas competitivas en costes y diferenciación respecto a las seguidoras, que les permiten obtener mayores beneficios y cuotas de mercado, además, son sostenibles en el tiempo. Ser pionera en el mercado permite obtener una mayor cantidad de producción acumulada, reduciéndose así los costes unitarios del producto y permitiendo a la empresa una posición de liderazgo en costes respecto a los competidores. (LIEBERMAN \& MONTGOMERY, 1998)

Shepherd, Ettenson y Crouch $^{(4)}$ También observan la existencia de un efecto directo de la entrada temprana en la rentabilidad de las nuevas empresas y comprueban la existencia de un impacto positivo de la posición pionera en el crecimiento de la cuota de mercado.

\subsection{El Efecto del Entorno Sectorial de la Empresa Telefónica del Perú}

Existen factores del entorno sectorial que van a moderar el impacto de la estrategia pionera en la consecución y mantenimiento de ventajas competitivas, ya que van a definir el ambiente competitivo en el que se van a desenvolver las empresas. Cuanto mayor dinamismo se observa en el sector, menos posibilidades tienen las empresas pioneras de obtener una ventaja competitiva sostenible.

La entrada de Telefónica del Perú se dio en condiciones de estabilidad y de poco dinamismo, por lo que tuvo mayores posibilidades de obtener ventajas competitivas por no verse sustancialmente modificadas las condiciones que existían en el momento de su entrada al mercado.

Sarkar, Cavusgil y Aulakh analizan una muestra de empresas del sector de telecomunicaciones y observan que: a) el carácter oligopolista, b)las elevadas economías de escala y c) el importante grado de interdependencia estratégica; estos elementos favorecen el desarrollo de las habilidades de los pioneros para obtener beneficios estratégicos y económicos, generando barreras de entrada para posteriores competidores. (GARCIA VILLAVERDE \& RUIZ ORTEGA, 2006).

Otro aspecto del entorno que se analiza es el tipo de proceso regulatorio que se da en el país donde pretende entrar la empresa pionera. En este sentido, al

(4) Citados por VILLAVERDE en la tesis: "El Momento de Entrada en el Mercado y la Generación de Ventajas Competitivas Sostenibles". 
tiempo del ingreso de Telefónica, el organismo regulador tenía una normatividad que restringía la competencia, por lo que el desarrollo del sector se vio afectado por el contrato de concesión que cerró el mercado a potenciales competidores, lo que le permitió al primer entrante conseguir y sostener ventajas estratégicas y económicas. (GARCIA VILLAVERDE \& RUIZ ORTEGA, 2006)

\subsection{El Efecto de los Recursos y las Capacidades}

Lieberman y Montgomery señalan que el desarrollo de una estrategia pionera puede permitir acumular recursos y capacidades, que sean valiosos y difíciles de imitar aprovechando un mejor posicionamiento en el ámbito geográfico, tecnológico y de la percepción de los consumidores, durante un determinado período de liderazgo.

Un aspecto relevante en el análisis de los factores internos relacionados con la estrategia pionera, es la necesidad de las empresas de desarrollar barreras de entrada para obtener un beneficio superior (LIEBERMAN \& MONTGOMERY, 1988)

Las barreras de entrada, basadas en la consecución de recursos y capacidades superiores, ofrecen a los pioneros la posibilidad de actuar en la industria sin competencia durante un período determinado. Estas barreras pueden proporcionar a la empresa una situación de monopolio temporal, que permite a la empresa obtener unas ventajas competitivas e influir en el desarrollo del mercado para que sean sostenibles (GOLDER \& TELLIS, 1993).

Una amplia ventaja temporal puede permitir a la empresa establecer una fuerte identidad de marca y modificar los gustos de los clientes para adaptarlos a la oferta de la empresa (GARCIA VILLAVERDE \& RUIZ ORTEGA, 2006).

La empresa pionera podrá seleccionar y asegurar los canales de distribución y los proveedores, consiguiendo una elevada reputación ante los mismos, que difícilmente puede ser igualada por los seguidores. También puede obtener prestigio entre los clientes e incluso, moldear su estructura de costes.

Si los pioneros evolucionan favorablemente desde su posición inicial, pueden desarrollar fidelidad de los clientes a su producto por su experiencia acumulada o convertir su producto en el estándar del sector en relación al diseño o la tecnología.

En definitiva, esta ventaja temporal proporciona a las empresas pioneras la oportunidad de establecer unos mayores precios o bien alcanzar una ventaja en costes, como consecuencia de este período de monopolio. Estas ventajas 
pueden ser sostenibles y constituir una fuerte barrera de entrada, si se produce un aprendizaje acumulativo basado en los recursos y en las competencias distintivas de la empresa.

\section{Análisis económico de la telefonía movil en el Perú}

En el año 2013 se promulgaron numerosas leyes que erosionaron las ventajas competitivas de la empresa pionera, lo cual incentivó el ingreso de nuevas operadoras y fomentó la competencia entre las empresas que prestan el servicio público de telecomunicaciones. En ese sentido el Texto Único Ordenado de la Ley de Telecomunicaciones - TUO fomentó la libre competencia en la prestación del servicio público de telecomunicaciones mediante el control y regulación por parte del OSIPTEL. Asimismo, en febrero de 2013, mediante Resolución Ministerial N 091-2013MTC/03, se renovó el contrato de concesión con Telefónica Móviles, esta vez el Estado negoció con la empresa operadora un plan de ampliación de la cobertura a 409 capitales de distrito, con ello el Estado garantizó el acceso de todo peruano a la telefonía Móvil, además de la creación de una Tarifa Única Social, cuyo coste está por debajo del establecido por Ley como tarifa mínima ${ }^{(5)}$ para zonas consideradas con pobreza y extrema pobreza.

La promulgación de este dispositivo ha sido el reflejo de una oportuna intervención del Estado para promover el subsidio cruzado de los servicios de red, creando una obligación para la empresa privada de invertir en zonas que no son rentables para las empresas privadas.

Con la aprobación del Decreto Supremo Nº16-2013-MTC, se establecieron medidas complementarias para la portabilidad numérica y los cambios que experimentaron los usuarios de los servicios públicos de telecomunicaciones fueron la reducción del plazo para el procedimiento pasando de siete días a un máximo de 24 horas, se estableció la continuidad del servicio, estableciéndose un plazo máximo de interrupción de tres horas, y la ventana de cambio sería entre as 00.00 y las 6:00 horas.

Con este nuevo procedimiento los usuarios pueden cambiar de operador a través de un mecanismo muy sencillo y en corto plazo, con ello, sí desean cambiar de operador de uno a otro que le ofrezca mejores condiciones, el procedimiento de portación ya no es un obstáculo.

Estas nuevas condiciones ofrecieron a los concesionarios mayores incentivos a crear estrategias pro portabilidad. El efecto ha sido que más de 3 millones de usuario hayan decidido cambiar de operador desde la vigencia de esta norma.

S/. 0.23 (0/23 Centavos de Sol). Equivale a menos de un centavo de dólar. 
Otra Ley que ha tenido un impacto en el mercado, es la Modificación al Reglamento General de Tarifas, mediante esta norma se estableció distintos tipos de tarifas distinguiendo las tarifas establecidas y promocionales, la norma señala que al momento de la facturación las empresas operadoras deben señalar expresamente el costo de cada una. Asimismo se estableció un plazo máximo de 180 días para aplicación de tarifas promocionales.

El impacto de esta norma, se ve reflejada en la información específica que reciben los usuarios, pues ahora efectivamente saben cuánto pagan por cada servicio contratado, se consiguió cambiar la oferta comercial de las empresas operadoras al establecer un plazo máximo de permanencia de una oferta.

En octubre del 2014 se publicó la Resolución del Consejo Directivo $N^{\circ}$ 123-2014-CD/OSIPTEL - Nuevo Reglamento de Calidad. Con esta norma se establecieron indicadores del servicio móvil de voz, calidad de cobertura del servicio, disponibilidad de los servicios finales y se estableció un tiempo límite de interrupción de servicio por un espacio de 90 minutos para Lima y el Callao y 180 minutos para las demás provincias.

Con la aplicación de esta norma el Estado creo desincentivos para la ocurrencia de eventos críticos de amplia duración.

Con las Modificaciones al Texto Único Ordenado de las Condiciones de Uso, se reguló mediante Resolución de Consejo Directivo № 138-2014-CD/OSIPTEL, el desbloqueo de Equipos Terminales Móviles, por tanto se prohibió la venta de equipos terminales que tengan restricción de acceso a la red de otro operador.

Asimismo, con esta norma se estableció la prohibición del condicionamiento de la contratación de servicios móviles a plazos forzosos, de esta manera todos los contratos de servicios móviles serán siempre a plazo indeterminado y se estableció contratos adicionales para la provisión del equipo terminal. De esta manera quedó clara la distinción entre la celebración de contratos para la adquisición equipos terminales y para la provisión del servicio. Esto a raíz de que las empresas ofrecían planes a plazo forzoso de un año como mínimo para la adquisición de un equipo, así los usuarios pagaban el equipo y el servicio por un año, durante este periodo los usuarios no podían resolver el contrato, tampoco podían cambiarse de operador, la terminación anticipada del contrato estaba sujeta al pago de una penalidad muy alta. Sin embargo con la nueva legislación, el pago de la penalidad estará sujeto al empleo de una fórmula, haciendo predecible el pago de la penalidad y distinguiendo el precio por la adquisición del equipo terminal y por la prestación del servicio.

Las falencias de la autorregulación hicieron necesario que en el año 2004 se determine y diseñe nuevos cargos móviles por interconexión, así se estableció 
tarifas aplicables hasta diciembre del año 2017. Haciendo una distinción entre empresas establecidas (Telefónica del Perú SAA y Telefónica Móviles) y empresas con reducida participación en el mercado. De esta manera se fijó US\$ 0.0179 para el primer grupo y US\$ 0.0201 para el segundo grupo.

Esta norma significa un incentivo para las empresas con poca participación en el mercado para incrementar sus niveles de cobertura.

El reglamento de la Ley para el fortalecimiento de la Expansión de Infraestructura en Telecomunicaciones estableció un régimen especial y temporal para la instalación y expansión de los servicios públicos de telecomunicaciones. Así el procedimiento de obtención de autorización para la instalación de infraestructura se sujeta a un procedimiento de aprobación automática.

Esta serie de normas promulgadas en los años 2013 y 2014 han tenido un importante impacto y han logrado erosionar las ventajas competitivas de la empresa pionera, quien en la actualidad es la que pierde más suscriptores, pues al haber estado prestando el servicio en calidad de monopolio cerca de 11 años, no estaba preparaba para enfrentar un mercado más dinámico y competitivo.

Por otro lado las normas han generado incentivos para el ingreso de nuevas operadoras al mercado móvil peruano, toda vez que las empresas buscan seguridad jurídica para realizar sus inversiones, al aprobarse un régimen de aprobación automática para la instalación de infraestructura, las empresas ya no tienen que enfrentarse a una decisión incierta acerca de sus proyectos de infraestructura.

Otro efecto importante ha sido la proliferación de Operadoras Móviles, se anunció que para el 2018 se contará con cuatro nuevas operadoras móviles virtuales, y las cuatro operadoras tradicionales, se contará con ocho operadoras, gracias a la creación de esta figura que permite operar sin la necesidad de instalar infraestructura, sino arrendarla.

Es así como a través de una adecuada intervención estatal, mediante un organismo regulador especializado y con un marco normativo acertado se ha logrado impactar positivamente en un mercado que por las características que presentan, servicios de red, es difícil por si sólo fomentar competencia.

\section{Estrategias y acciones de las operadoras}

\subsection{Expansión de las Redes Privadas Móviles}

- Telefónica.- Fue la primera empresa en introducir el concepto de las redes privadas en el Perú, al lanzar en el año 2006 su servicio denominado "Red Privada Movistar " (RPM), que consistía en 
otorgar una bolsa de minutos libres para realizar llamadas hacia otras líneas de la RPM marcando numeral (\#) y un el código RPM asociado a la línea que se deseara contactar.

- $\quad$ América Móvil.- Como respuesta a servicio de RPM de Telefónica, lanzó en julio de 2006 la "Red Privada Claro Empresas (RPC e) únicamente para clientes corporativos empresariales. Posteriormente, en noviembre 2010, lanzó su familia de planes RPC para el público postpago en general.

- $\quad$ Entel.- En octubre de 2014, incluyó, como atributo de algunos de sus planes postpago, el servicio de Red Privada Entel (RPE) que otorgaba minutos ilimitados para realizar llamadas hacia móviles de Entel tanto postpago como prepago y fijos nacionales.

- Viettel.- Desde su ingreso al mercado en julio de 2014, lanzó también planes con el servicio denominado "Red Privada Bitel" (RPB) que consiste en otorgar un bono de minutos libres para llamadas a todos los postpago de Bitel.

\subsection{Acceso a Redes Sociales Ilimitadas}

- $\quad$ Telefónica.- El beneficio consistía en otorgar a los clientes de altas nuevas de planes postpago comercializados entre abril y mayo de 2013, un bono temporal para hacer uso de "Facebook Ilimitado" por un periodo de hasta de 3 meses.

- $\quad$ América Móvil.- Comenzó a ofrecer el servicio de WhatsApp gratis hacia fines de 2014 como beneficio adicional para altas nuevas de los planes "RPC Ilimitado". Posteriormente, en febrero de 2015, extendió este beneficio para clientes de la competencia que se portaran hacia los planes "Conexión Plus".

- $\quad$ Entel.- Por su parte, desde agosto de 2015 ofrece por S/. 1 el servicio de WhatsApp ilimitado por un día para determinados clientes control (actuales y altas nuevas) y prepago;y la promoción "Superyapa Potenciada Prepago" que otorga 30 minutos todo destino y WhatsApp ilimitado para aquellos clientes prepago que realicen una recarga mínima de $S / .5$, con una vigencia de 3 días.

- $\quad$ Viettel.- Ofrece el servicio de WhatsApp ilimitado para los clientes de sus planes prepago "Bifri" (desde enero de 2015), "Ahorra", 
"Ganga" y "Nuevo UNI2" (dese marzo de 2015), siempre que realicen como mínimo una recarga mensual de S/. 3 .

\subsection{Planes con Servicios Ilimitados}

Entel acompañó el inicio de sus operaciones comerciales lanzando los planes "Entel Libre" (en octubre de 2014) y "Entel Control" (en noviembre de 2014), ambos con rentas de S/. 199.00, los cuales tenían la novedad de otorgar, dentro del plan además de otros servicios, minutos ilimitados para realizar llamadas locales a todo destino.

- Telefónica respondió en mayo de 2015, otorgando el mismo beneficio a los clientes que contratasen los planes "Vuela" (con acuerdo de adquisición) y "Vuela Ahorro" (sin acuerdo de adquisición) con rentas mensuales desde S/. 140 y S/. 125, respectivamente.

A-Móvil también incluyó este beneficio desde junio de 2015, con el lanzamiento de su plan "Conexión" (con acuerdo de adquisición) con rentas desde S/. 139. Posteriormente, en julio de 2015, extendió este beneficio a clientes que contratasen el plan "Conexión Chip" (sin acuerdo de adquisición) con renta mensual de S/. 99.

Por su parte, Viettel, ofrece, desde junio de 2015, planes postpago desde S/. 29.90 que incluyen Internet ilimitado; $y$, desde agosto de 2015 un bono de Internet ilimitado por 12 meses para aquellos clientes que adquieran de forma conjunta un plan prepago y un equipo Smartphone.

\subsection{Lanzamiento del servicio de internet con tecnología 4G}

El 22 de julio de 2013 los postores T-Móviles y Americatel (filial del grupo chileno Entel) fueron los primeros en lanzar planes postpago con tecnología 4G LTE (en adelante, planes 4G) fue Telefónica, quien en noviembre de 2013 empezó la pre-venta de sus planes 4G denominados "Vuela", habilitando el servicio de Internet 4G LTE desde el primero de enero de 2014.

A pesar de no haberse adjudicado ningún bloque de la referida subasta, la segunda empresa en brindar el servicio de Internet 4G LTE fue A-Móvil, en mayo de 2014, a través de los planes "Conexión" y "Conexión Plus". Para ello, realizó modificaciones 
técnicas, con el objetivo de habilitar la red 4G sobre la banda 1900 $\mathrm{MHz}$ que posee.

Por último, Entel inició la comercialización de planes 4G en octubre de 2014 (fecha de inicio de operaciones comerciales bajo la marca Entel), a través de los planes "Entel Libre" y "Entel Control".

El lanzamiento de los nuevos planes 4G no solo significó el incremento de las velocidades para los clientes postpago, sino que también vino acompañado de un incremento de velocidades para los clientes prepago. Además, trajo consigo mayores atributos entre los que destacan el acceso a aplicaciones gratuitas y el incremento de las capacidades de transmisión de los paquetes de datos.

\subsection{Comercialización de Equipos Terminales}

Hasta el año 2014, la estrategia comercial adoptada por los operadores móviles era la de comercializar planes postpago con acuerdos de adquisición de equipos móviles. De esa forma, a mayor plazo forzoso y/o renta mensual del plan, se podía obtener un mayor descuento sobre el precio de venta del equipo.

Si bien la referida estrategia fue adoptada también por Viettel y Entel desde el inicio de sus operaciones comerciales, Entel, aprovechando el relanzamiento de la portabilidad numérica móvil, empezó a ofrecer descuentos adicionales en los equipos a clientes de la competencia que decidieran portarse a un plan postpago. En respuesta a ello, los demás operadores redujeron los precios de sus equipos, haciendo que incluso equipos de alta gama tuvieran un precio de S/. 1 asociados con determinados planes de altas rentas mensuales.

Adicionalmente, lanzó el "Programa Inteligente" el cual permitía renovar anualmente el equipo terminal por la última versión disponible en el mercado, para aquellos clientes que contrataran el Plan "Vuela Inteligente" cuya renta mensual es S/. 199.90 . Por su parte, en julio de 2015, A-Móvil desarrolló un nuevo esquema de adquisición de equipos terminales, el cual permite financiar el importe de los equipos terminales hasta en 24 cuotas mensuales. 
Tabla Nro.2. Porlabilidad Acumulada Neta

\begin{tabular}{|c|c|c|c|c|c|c|c|}
\hline \multirow{2}{*}{\multicolumn{2}{|c|}{$\begin{array}{l}\text { Líneas Móviles portadas } \\
\text { Del } 16 \text { de julio de } 2014 \\
\text { al } 31 \text { de marzo de } 2018\end{array}$}} & \multicolumn{5}{|c|}{ Operador Receptor (Gana Clientes) } & \multirow{2}{*}{$\begin{array}{l}\text { Total de } \\
\text { Líneas } \\
\text { Perdidas }\end{array}$} \\
\hline & & e) entel & & M movistar & Bbitel & Ânka & \\
\hline \multirow{5}{*}{$\begin{array}{l}\text { Operador } \\
\text { Cedente } \\
\text { (Pierde } \\
\text { Clientes) }\end{array}$} & e) entel & $\mathrm{x}$ & 773,272 & 577,434 & 86,826 & 3517 & 1441,049 \\
\hline & 0 & 1212,100 & $\mathrm{x}$ & 868,407 & 159,205 & 5192 & 2244,904 \\
\hline & Mimovistar & 1587,994 & 1417,637 & $\mathrm{x}$ & 193,389 & 4254 & 3203,274 \\
\hline & Bbitel & 228,551 & 305,020 & 240,061 & $\mathrm{x}$ & 2525 & 776,157 \\
\hline & Ânka & 3309 & 7978 & 1648 & 1529 & $\mathrm{x}$ & 14,646 \\
\hline \multicolumn{2}{|c|}{ Total de líneas ganadas } & 3031,954 & 2503,907 & 1687,550 & 15,488 & 15488 & 7679,848 \\
\hline
\end{tabular}

Fuente: Información reportada por las empresas. Elaboración Propia

\section{Conclusiones}

5.1. Hemos analizado los instrumentos legales promulgados a partir del año 2013 y hemos podido comprobar que el nuevo marco regulatorio impacto positivamente el mercado móvil, generando competencia entre las cuatro operadoras, y ha fomentado que las empresas entrantes incrementen su participación en el mercado, erosionando la ventaja que tenía Telefónica del Perú como empresa pionera.

5.2. En el corto plazo, se espera que tanto Entel como Viettel sigan ganando participación, y se espera también el ingreso de los Operadores Móviles Virtuales. Con ello, se presentará mayor competencia y desconcentración en el mercado. Producto de ello, se espera que los operadores lancen más ofertas comerciales que beneficien a los usuarios y dinamicen el mercado, como las que se han venido observando en los últimos meses. Del mismo modo, se espera que los servicios móviles se sigan expandiendo en más distritos del país.

5.3. Las empresas entrantes se vieron favorecidas por el marco regulatorio que fomento la competencia entre las mismas, y el ganador neto fue el consumidor. En la actualidad hay registradas 110 líneas por cada 100 habitantes haciendo un contraste con los años 1993, cuando por cada 100 habitantes solo 1 accedía a este servicio.

5.4. En cuanto a uno de los efectos de las dinámicas competitivas entre la operadoras, se tiene que a julio de más de 6 millones de personas migraron de operadoras y la perdieron neta fue movistar que perdió 3 203, 274 líneas. 


\section{Bibliografía}

CÁCERES BARRANTES, R. (2005). “La Regulación para el desarrollo de las Telecomunicaciones en Perú 1993-2001". JCAS Ocasional Paper Nº 25.

GARCIA VILLAVERDE, G y L. RUIZ ORTEGA (2016). Marker Share Reward to pioneering Brands: As Empirical Analysis and Strategic Implications, Management Since.

KERIN, R., VARADARAJAN, R. R., \& PETERSON, R. (1992). "First-mover advantage: A synthesis, conceptual framework, and research propositions, Journal of Marketing". KRESALJA, R., \& OCHOA CARDISH, B. (2009). Derecho Constitucional Económico. Lima: Fondo Editorial de la Pontificia Universidad Catolica del Perú.

Ley 30083-Ley que Establece Medidas para Fortalecer la Competencia en el Mercado Móvil. Obtenido de OSIPTEL: https://www.osiptel.gob.pe/articulo/ley-30083-fortalecer-competencia-servicios-moviles

Ley de Telecomunicaciones y su Reglamento -Decreto Supremo $N^{\circ}$ 20-2007MTC. Obtenido de: http://transparencia.mtc.gob.pe/idm docs/normas legales/1 0 2137.pdf

LIEBERMAN, M., \& MONTGOMERY, B. (1998), "First movers advantages". Strategic Management Journal.

MIRANDA BALDEON, C. (2014). La Autorización de Servicio Público. Bases para una Liberalización Plena del Sector de Telecomunicaciones. Lima: Fondo Editorial de la Pontificia Universidad Católica del Perú.

OSIPTEL. Obtenido de Libro Blanco sobre la apertura de las telecomunicaciones en el Perú: www.osiptel.gob.pe

Resolución de Consejo Directivo Nº24-2014-CD/OSIPTEL. Obtenido de OSIPTEL: https://www.osiptel.gob.pe/Archivos/ResolucionAltaDireccion/ConsejoDirectivo/ Res024-2014-CD.pdf

Resolución de Consejo Directivo № 123-2014-CD/OSIPTEL. Obtenido de OSIPTEL: https://www.osiptel.gob.pe/Archivos/ResolucionAltaDireccion/ConsejoDirectivo/ Res123-2014-CD.pdf 